\title{
Morbilidad y Mortalidad de Recién Nacidos con peso de nacimiento inferior a 1.500 gramos
}

\author{
Dra. Elizabeth Weldt S. ${ }^{\text {; }}$ Dr. Jaime Alarcón R. ${ }^{1}$; Dr. Sergio Vaisman W.1-2 \\ Morbility and Mortality in 121 Infants with \\ Birth Weight Less Than $1.500 \mathrm{Grams}$
}

\begin{abstract}
Morbility and mortality among 121 infants with birth weights less than 1.500 grams born during year 1982 were studied. Jaundice was present in $54,6 \%$ of the cases and was followed in incidence by metabolic problems (hypoglicemia, hypocalcemia, late metabolic acidosis) and respiratory distress syndrome. Forty seven infants survived $(39 \%)$. There were no survivors when birth weight was less than 750 grams. Survival rates were respectively, $23,6 \%$ for infants weighting 751 to 1.000 grams: $40 \%$ between 1.001 and 1.250 grams and $63,3 \%$ between 1.250 and 1.500 grams. The principal causes of death nere extreme prematurity, respiratory distress syndrome and intracraneal bleeding. This type of study is necessary in Neonatal Units to plan adecuate resources and to get the best possible results.
\end{abstract}

La mortalidad de los recién nacidos de bajo peso de nacimiento ha disminuido desde el inicio de los cuidados intensivos neonatales ${ }^{1} \cdot 2 \cdot 3-4$ 5. Países desarrollados como Estados Unidos y Canadá publican aumentos muy significativos de la sobrevida de los Recién Nacidos con pesos de $1.500 \mathrm{~g}$. o menos, en relación a los avances en los cuidados obstétricos y neonatales que comenzaron en la década del 701 . En forma paralela se mejoró la calidad de la sobrevida de los Recién Nacidos de Pretérmino5.

En nuestro medio hemos observado también en los últimos años un aumento en las expectativas y condiciones de vida en los Recién Nacidos de bajo peso de nacimiento. Esto nos ha motivado a estudiar este grupo de riesgo con el fin de conocer la morbilidad más frecuente que les afecta $y$ las causas de muerte, para continuar progresando en su manejo.

\section{MATERIAL Y METODO}

Durante el ă̄o 1982 nacieron en la Materni. dad del Salvador 11.429 niños, 121 con peso de

${ }^{1}$ Unidad de Neonatología. Servicio de Obstetricia y Ginecologia. Hospital del Salvador. Servicio de Salud Metropolitano Oriente.

2 Departamento de Obstetricia y Ginecología. División Ciencias Médicas Oriente. Facultą̧ de Medicina de la Universidad de Chile. nacimiento inferior a $1.500 \mathrm{~g}$. , lo que da una incidencia de $1.06 \%$.

Los datos clínicos de estos 121 Recién Nacidos (RN) fueron registrados en una ficha computarizada, que consta de identificación del $\mathrm{RN}$, antecedentes familiares, del embarazo y parto, diagnósticos, tratamiento, condiciones al alta, días de estada y alimentación recibida.

La ficha se procesó en un computador IBM 370 de la Facultad de Ciencias Físicas y Matemáticas de la Universidad de Chile, empleando un lenguaje diseñado para asistir a la realización de análisis estadisticos (SPSS).

Los pacientes del estudio fueron agrupados según Peso de Nacimiento (PN), Edad Gestacional (EG) y Crecimiento Intrauterino. La EG se obtuvo por anamnesis (fecha de la última mens. truación) y por la evaluación pediátrica según el esquema de Dubowitz. Cuando no hubo concordancia entre ambas se denominó EG incierta. Según el crecimiento intrauterino los RN fueron clasificados en: pequeño, adecuado o grande para la EG, utilizando las curvas de Lubchenco.

La Morbilidad se clasificó en Ictericia, Infecciones, Sindrome de Dificultad Respiratoria (SDR), Problemas Hematológicos, Malformaciones Congénitas, Problemas Metabólicos, Trauma. tismos del Parto, Alteraciones del Sistema Nervioso Central (SNC), Apneas, Infección Conratal.

Las Ictericias se dividieron en Incompatibili. 
dad de grupo clásico $(\mathrm{AB} / \mathrm{O})$, In compatibilidad Rh e Hiperbilirrubinemia Idiopática.

Las Infecciones se clasificaron en Menores (Onfalitis, Conjuntivitis, Piodermitis, Rinitis), Mayores (Septicemia, Bronconeumonias, Meningitis, Osteoartritis) y Diarreas.

Los casos con Sindrome de Dificultad Respiratoria se ordenaron en: Sindrome de Dificultad Respiratoria Idjopática (SDRI), Taquipnea Transitoria (TT), Síndrome de Broncoaspiración y Bronconeumonia Connatal.

Entre los problemas hematológicos se consideraron los casos de anemia con valores de $\mathrm{Hb}$ que requirieron transfusión, los de Policitemia con Hematocrito superior a $65 \%$ y los síndromes hemorragicos.

Las Malformaciones Congénitas se clasificaron en los Sistemas preferentemente afectados.

En los problemas metabólicos se incluyeron los pacientes con hípoglicemia, hipocalcemia y acidosis metabólicas tardía del prematuro.

Los traumatisnos del parto abarcan las equimosis múltiples, fractura de clavícula, cráneo, húmero y fémur, céfalohematoma y hematoma subaponeurótico.

En las alteraciones del SNC se incluyeron las disfunciones neurológicas especificando su causa cuando la ultrasonografia, la autopsia 0 ambas lo hicieron posible.

\section{RESULTADOS}

La Tabla 1 muestra la distribución por PN. Destaca un $43 \%$ de $R N$ con PN inferior a $1.000 \mathrm{~g}$., lo que tiene importancia en la mortalidad giobal del grupo. El $88,4 \%$ del grupo total correspondió a RN adecuados para la edad gestacional.

\section{Tabla I.}

Distribución de $121 \mathrm{RN}$ con Peso de Nacimiento Inferior a $1.500 \mathrm{~g}$.

\begin{tabular}{ccc} 
PN & NO & $\%$ \\
$501-750$ & 18 & 14,9 \\
$751-1.000$ & 34 & 28,1 \\
$1001-1.250$ & 20 & 16,5 \\
$1.251-1.500$ & 49 & 40,5 \\
\hline Total & 121 & 100,0
\end{tabular}

Entre los antecedentes obstétricos de los pacientes estudiados había infección ovular en $31,9 \%$, en 10,6 se registró antecedente de hipertensión arterial. La metrorragia inició el parto prematuro en $7,07 \%$ y en $50 \%$ de las madres no hubo antecedentes mósbidos durante el embarazo.
Al analizar las condiciones al nacer mediante la puntuación de Apgar, se encontró que 16,8\% de estos RN falleció en los primeros 5 minutos de vida (Apgar 0 a los 5'). Todos pertenecían al grupo de $\mathrm{PN}$ inferior a $1.000 \mathrm{~g}$.

En la Tabla 2, vemos que el $54,6 \%$ de los RN estudiados presentan ictericia, en este grupo predominó la ictericia idiopática con 59 casos, $3 \mathrm{RN}$ presentaron incompatibilidad de grupo clásico y uno incompatibilidad $\mathrm{Rh}$. Todos fueron tratados con Fototerapia por un promedio de 5,86 días (rango 1 a 15 dias); en 16\% de estos RN se requirió el empleo de transfusión de recambio.

Tabla 2.

Marbilidad de 121 RN con PN inferior a $1.500 \mathrm{~g}$.

$\begin{array}{lrr} & \text { N } & \% \\ \text { Ietericia } & 66 & 54,6 \\ \text { Problemas Hematológicos } & 49 & 40,5 \\ \text { S.D.R. } & 47 & 38,8 \\ \text { Apneas } & 34 & 28,0 \\ \text { Malformaciones Congénitas } & 25 & 20,7 \\ \text { S.N.C. } & 24 & 19,8 \\ \text { Problemas Metabólicos } & 21 & 17,3 \\ \text { Diarreas } & 18 & \mathbf{1 4 , 9} \\ \text { Infecciones Menores } & 17 & \mathbf{1 4 , 0} \\ \text { Infecciones Mayores } & 15 & \mathbf{1 2 , 4} \\ \text { Traumatismos del Parto } & 6 & \mathbf{4 , 9} \\ \text { Infección Connatal } & 2 & 1,6\end{array}$

Los problemas hernatológicos ocuparon el segundo lugar con 49 casos, de los cuales 44 presentaron anemia y requicieron transfusión, 7 RN tuvieron policitemia y en 4 fue necesario hacer eritroféresis.

EI S.D.R. ocupa el tercerce lugar con 47 RN. En 28 de ellos se diagnosticó S.D.R.I., 12 tuvieIon TT, 6 Bronconeumonia y 1 Síndrome Aspirativo. En este grupo fallecieron 25 RN y el $50 \%$ de ellos turo un PN menor a $1.000 \mathrm{~g}$.

Los episodios de Apnea ocuparon un lugar importante en nuestra serie. En la mayoría fue secundaria, (26 RN); sólo 8 casos fueron catalogados de apnea primaria.

En 25 RN se diagnostico alguna malformación congénita, en su mayoría fueron defectos cardiovasculares, incluyendo la persistencia del ductus arterioso.

Las alteraciones del S.N.C. ocurrieron en 24 RN, incluyendo 22 casos con hemorragias intracraneanas, un caso de disfunción neurológica de causa no identificada y otro con sindrome convulsivo. De los niños que suftieron hemorragias intracraneanas fallecieron 13.

Los trastornos metabólicos ocurrieron en 21 pacientes. En 16 acidosis metabólica tardía del prematuro, en 6 hubo hipoglicemia y en 4 alte. 
raciones de los electrolitos plasmáticos, (algunos nifios presentaron más de un trastorno simultáneamente).

Las Diarreas, que ocupan una proporción importante de la morbilidad correspondieron a una epidemia por E. Coli cepa 0142 que ocurrió en el servicio entre Enero y Agosto de $1982^{11}$.

Se encontraron infecciones menores en 17 RV, en 11 onfalitis, en 3 conjuntivitis, en 2 piodermitis y uno con rinitis. El agente causal predominante fue el Staphylococcus Aureus.

Hubo 15 pacientes que sufrieron infecciones mayores, en 8 septicemias, 6 bronconeumonias $y$ uno con infección urinaria. Los agentes causales fueron Klebsiella y Pseudomona Aeriginosa.

Seis RN tuvieron equimosis múltiples debido a partos traumáticos.

Se diagnosticaron 2 RN con infecciones connatales, una toxoplasmosis y una listeriosis.

La Figura I, muestra que no hubo sobrevidas cuando el peso de nacimiento era inferior a $750 \mathrm{~g}$. En el grupo comprendido entre $750 \mathrm{y}$ $1.000 \mathrm{~g}$. sobrevivió el $23,6 \%$, entre $1.001 \mathrm{y}$ 1.250 g. vivió el $40 \%$ de los RN y entre 1.251 y $1.500 \mathrm{~g}$, el $63,3 \%$.

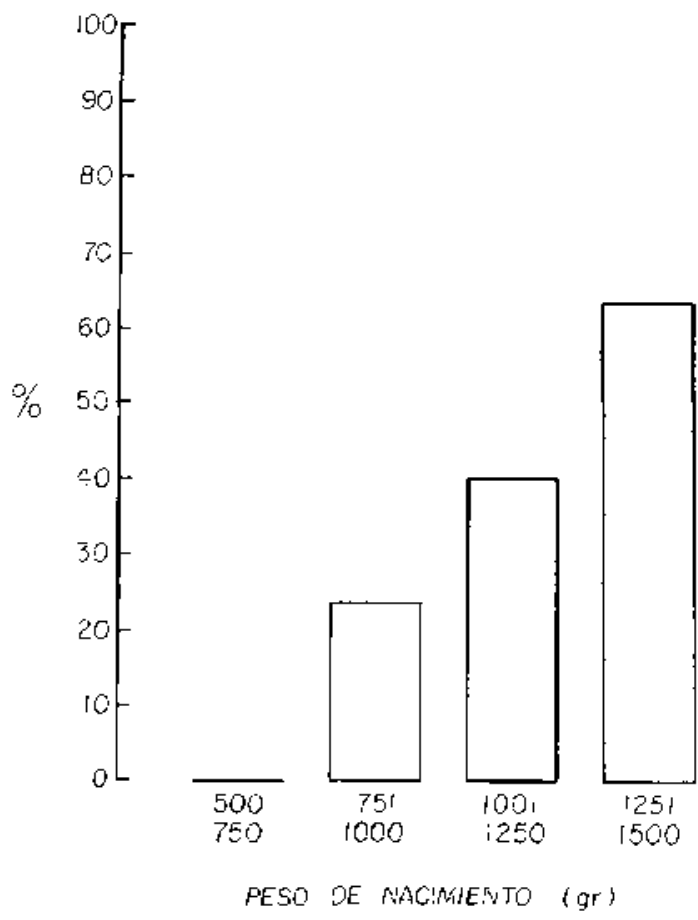

Figura 1

Sobrevida de $12 \mathrm{t}$ tecien nacidos según geso de nacimiento

La Tabla 3 muestra la causa de muerte en 74 RN. El primer lugar está dado por prematurez extrema, diagnóstico que se aplicó a los RN con PN inferior a $1.000 \mathrm{~g}$. incapaces de mantener espontáneamente la respiración. Le siguen en frecuencia el S.D.R. con 17 casos y la hemorragia intracraneana con $13 \mathrm{RN}$, este último diagnóstico se efectuó por Ecografía encefálica y/o Autopsia.

Tabla 3 .

Causa de muerte en 74 RN con Peso de Nacimiento Inferior a $1.500 \mathrm{~g}$.

Causa de Muerte

No

Prematurez Extrema

Síndrome de Dificultad Respiratoria S.D.R.I.

Atelectasia Pulmonar

Hemorragia Pulmonar

Sindrome Aspirativo

Hemorragia Intracrancana

Malformaciones

Malformaciones Múltiples

Onfalocele

Microcefalia

Acraneo

Agenesia Diafragmática

Septicemia

Hidrops por Incompatibilidad R1

Asfixia Nenonatal Grave

TOTAL

10

4

2

7

2

1

1

1

6

2

1

\section{DISCUSION}

Los RN con peso inferior a $1.500 \mathrm{~g}$. ocurren según nuestros datos con una incidencia de 10,58 por cada 1.000 nacidos vivos, superior a las tasas norteamericanas ${ }^{1 \cdot 6}$, de hace 10 arios $\left(9,48^{\circ} \%\right.$ oo). La intensa labor de prevención del parto prematuro ha reducido en forma muy significativa la incidencia en los paises desarrollados ${ }^{6}$, a diferen. cia de 10 que ocurre en nuestro pais, en que todavía es posible esperar Recién Nacidos de muy bajo peso como consecuencia de abortos provocados, embarazos no controlados, infecciones ovulares y partos espontáneos de origen traumático. En esta revisión $50 \%$ de los Recién Nacidos de bajo peso se relacionaban con problemas maternos capaces de desencadenar parto prematuro.

La ictericia aparece como la morbilidad más frecuente en nuestra población, sin embargo, todos los Recién Nacidos vivos requirieron de fototerapia, los demás fallecieron precozmente antes de mostrar ictericia.

El Síndrome de Dificultad Respiratoria fue la segunda causa de morbilidad. Creemos, sin embargo, que si se hubiera utilizado un manejo agresivo en la atención inmediata del $\mathrm{RN}$ como el propuesto por $\mathrm{Hirata}^{7}$, una porción significativa 
de los RN fallecidos en los primeros minutos de vida habría requerido ventilación asistida. Si además se considera que $28 \%$ de los recién nacidos sufrió uno o más episodios de apnea en los primeros días de vida requiriendo algún tipo de asistencia ventilatoria por esta causa, los RN con algún tipo de trastorno respiratorio se aproximan a $80 \%$.

La Hemorragia Intracraneana es una complicación importante en los recién nacidos de muy bajo peso de nacimiento, se documentó en 22 casos con Ecografía y se confirmó en Anatomía Patológica en 13.

La elevada incidencia de infecciones bacterianas intrahospitalarias se explica por la gran instrumentación a que son sometidos los recién nacidos de muy bajo peso; en el año estudiado sumaron 18 casos de Diarrea por E. coli Enteropatógeno Cepa 0142 originados en una infección intrahospitalaria de carácter epidémico ${ }^{11}$.

En la actualidad, en los países de mayor desarrollo se espera una sobrevida de $80 \%$ a $85 \%$ en los recién nacidos con peso de nacimiento entre 1.000 y 1.500 g. y de alrededor del $50 \%$ para pesos entre 800 y $1.000 \mathrm{g.}^{2-4}$; nuestras limitaciones técnicas no nos han penmitido por el momento alcanzar esos niveles. Aunque se discute si sería ético ocupar los recursos de atención intensiva en pacientes de menos de $750 \mathrm{~g}$. por las escasas expectativas de sobrevida y el mayor riesgo de secuelas ${ }^{6-8-9}$, publicaciones recientes muestran sobrevidas de 20 a $25 \%$ con seguimiento a mediano plazo muy satisfactorios $1-5-8-10$.

No conocemos cifras nacionales previas de mortalidad en recién nacidos con peso inferior a $1.500 \mathrm{~g}$. que nos permitan comparar si efectivamente hemos disminuido su mortalidad. Estudios como este en cada Servicio de Neonatología permitirán conocet los grupos de mayor mortalidad y la patología más frecuente con el objeto de buscar mejores resultados.

\section{RESUMEN}

Se estudian 121 RN con PN inferior a $1.500 \mathrm{~g}$., nacidos en la Maternidad del Salvador el año 1982 .

La morbilidad más frecuente fue la ictericia con un $54,6 \%$, le siguen los problemas metabólicos y el S.D.R.
La mortalidad global del grupo fue de $61,2 \%$ con una mortalidad del $100 \%$ para el grupo con $\mathrm{PN}$ inferior a $750 \mathrm{~g}$.

La primera causa de muerte fue prematurez extrema, le siguen en frecuencia el SDR y luego la Hemorragia Intracraneana.

\section{AGRADECIMIENTOS}

Se agradece la asistencia computacional a las señoritas Evelyn Sandoval P. y Maricel Sandoval P., y la dactilografia a la señorita lsabel Gamboa $\mathrm{H}$.

\section{REFERENCIAS}

I Saigal S., Rosenbaum P., Stoshopf B. " al: Follow up of infants $500-1.500$ \&. birth rejot: delivered to residents of a geograpicically defined region with perinatal intensive care facilities. J. Pediatr. 100: 606,1982 .

2 Hernández S.A., Lazarte R.A., Goldson E., et al. Mortaity and early morbidity in infants less than $1.000 \mathrm{~g}$. birth weight: Clin. Res, 29: $118 \mathrm{~A}, 1981$

${ }^{3}$ Koops B.L., Morgan L.J., Battagtio F.C.: Neonatal Mortality Risk in relation to birth weight and gestational age: J. Pediatr. 101: 969, 1982.

4 Pape K.E., Buncic R.J., A shby'S., Fitzhardinge P.M.: The status at two years of Low-birth weight infants born in 1974 with birth weight of less than $1.001 \mathrm{~g}$. 3. Pediatr. 92: 253, 1978.

5 Ment L.R., Scott D.T., Ehrenkranz R.A., Rothman S.S., Ducan C.C., Warshon J.B.: Recién Nacidos con peso de nacimiento igual o menor a $1.250 \mathrm{~g}$. Valora. ción prospectiva de su desarrollo neurológico durante el primer ario de vida postérmino. Pediatrics (Ed. Española) 14: 136, 1982.

${ }^{6}$ Horwood S.P. Boyle M.H., Torrance G.W., Sinciair J.C.: Mortality and Morbility of 500 to $1.499 \mathrm{~g}$. birth weight infants live-born to tesidents of a defined Geographic Region before and after Neonatal Intensive Care. Pediatrics 69: 613, 1982.

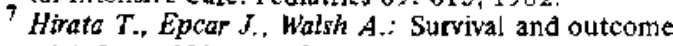
of infants 501 to $750 \mathrm{~g}$ : a six year experience. J. Pediatr. 102: 741, 1983

8 Bennett $F C_{,}$, Robinson $N, M$, Sells $C . J$ : Crecimiento y desarrollo de los nin̄os con peso de nacimiento inferior a $800 \mathrm{~g}$. Pediatrics (Ed. Española) 1S: 174, 1983.

${ }^{9}$ Britton S.B., Chin B., Fitzhardinge P.M. et al: Is intensive care justified for infants weighthing less than 801 g. at birth? J. Pediatr, 99: 937, 1981.

10 Hack M., Fanoroff A.A., Markatz: The low birth weight infant. Evaluation of changeing, N. Engl. $J$. Med. $301: 162,1974$.

11 Hering E., Marambio E., Prado $V_{.:}$Análisis de uл brote de diarrea por E. coli 0142 en recién nacidos. Rev. Chil. Pediatr. 55: 175, 1984. 